\title{
Influencia del Programa Comportamiento Seguro en los Trabajadores de Planta Callao -CLSA, Lima-Perú
}

CÉsar Rodríguez Del CARPio ${ }^{1}$

Recibido: 03/03/2020 AcEPTAdo: 17/09/2020 Publicado: 31/12/2020

\section{RESUMEN}

Los programas de seguridad basados en el comportamiento se aplican con la finalidad de reforzar el nivel de seguridad en el trabajo, pero si se hace por cumplir con un número de observaciones de comportamiento seguro establecidas en la meta del mes en el lugar de trabajo, genera en los trabajadores que solo cumplan con la seguridad mientras se les está observando 0 que los observadores reporten comportamientos que realmente no vieron; en consecuencia, los accidentes con trabajadores continúan y peor aún con casos fatales, los observadores dejan de creer en el programa de seguridad que están utilizando y la dirección llega a creer que con un mayor número de observaciones se logra corregir los comportamientos inseguros; por ello es que los resultados del programa no se alcanzan con el tiempo. Esto es consecuencia de no haber entendido los principios de la teoría de la seguridad basada en el comportamiento desde el inicio de su implementación. En la presente investigación se aplicó la teoría de la seguridad basada en los comportamientos para definir la tarjeta de observadores y para realizar las intervenciones con refuerzo positivo de manera eficiente. Así, el estudio se orientó a encontrar si hay diferencia significativa entre las observaciones de comportamiento seguro antes que el observador realice la retroalimentación con refuerzo positivo y las observaciones después de cada intervención, para ello se tomó sólo el número de observaciones seguras con el propósito de conocer el efecto que tiene la retroalimentación con refuerzo positivo que se da en la observación de comportamientos seguros en el periodo de estudio. Se encontró que hay diferencia significativa en los comportamientos seguros después de realizar la retroalimentación para un nivel de confianza del $95 \%$ y una reducción del $85.7 \%$ de accidentes incapacitantes en el periodo de estudio.

Palabras clave: Programa de Seguridad Basado en el Comportamiento (SBC); liderazgo y reducción de accidentes.

Influence of the Safe Behavior Program on the Workers of the Callao Plant -CLSA, LIMA-PERU

\section{ABSTRACT}

Behavior-based safety programs are applied with the aim of reinforcing the level of safety at work, but if it is done by complying with a number of observations of safe behavior established in the goal of the month in the workplace, it generates in workers who only comply with safety while being observed or that observers report behaviors that

\section{INTRODUCCIÓN}

En el Perú, de acuerdo a los Reportes de Notificación de accidentes de trabajo del Ministerio de trabajo y promoción del empleo, según la actividad económica en el periodo 2011 al 2015, identifica a la industria manufacturera como el sector que encabeza la estadística de accidentes laborales a nivel nacional, manteniéndose encima de otras actividades como de minería, construcción y de transportes.

En el proceso de envasado de bebidas gaseosas en botellas de vidrio retornable se reportan accidentes con incapacidad laboral generados en las tareas tales como en la selección de envases que retornan del mercado, de envases con producto, de cajas plásticas, de plataformas de madera entre otros, también en las tareas de limpieza y sanitización por el uso de químicos para conservar el estado sanitario de los equipos de envasado; así mismo, al hacer correctivos en las máquinas de las líneas de producción o al realizar el mantenimiento autónomo de los equipos. En éstas tareas existen peligros tales como proyección de objetos, trabajos cerca o con máquinas en movimiento, trabajos con objetos punzocortantes, atrapamientos, caídas a desnivel, contacto con materiales peligrosos, contacto con superficies calientes, contacto con energía eléctrica, etc. Los peligros identificados están siempre presentes; si no se siguen correctamente los procedimientos seguros de operación o no se usan adecuadamente los equipos de protección personal, podrían generar un accidente.

Son varios los factores que explican por qué la gente se accidenta, Villalobos (2018) explica que por el lado de la supervisión, muchas veces interesa el cumplimiento de las tareas; la ejecución de la tarea más sencilla tendrá un criterio de prevención que no hay que descuidar, pero si un operario viene haciendo algo mal y si no se le dice nada pensará que lo está haciendo de manera segura, esta misma situación de riesgo en otras circunstancias podría terminar en un accidente, pues si no ocurrió en la primera vez podría ocurrir en una siguiente oportunidad; aquí se resalta importancia que tiene el supervisor como líder de su equipo de trabajo en la percepción del riesgo, dependiendo

1 Ingeniero en Industrias Alimentarias de la Universidad Nacional Agraria - La Molina. Lima, Perú. Supervisor de Producción de CLSA

ORCID: https://orcid.org/0000-0003-2056-9619

E-mail: crodriguezd@lindley.pe 
they did not really see; consequently, accidents with workers continue and even worse with fatal cases, observers stop believing in the safety program they are using and management comes to believe that with a greater number of observations it is possible to correct unsafe behaviors; therefore, the results of the program are not achieved over time. This is a consequence of not having understood the principles of behavior-based security theory since the beginning of its implementation. In the present investigation, the behavior-based security theory was applied to define the observer card and to carry out positive reinforcement interventions efficiently. Thus, the study aimed to find out if there is a significant difference between the observations of safe behavior before the observer performs the feedback with positive reinforcement and the observations after each intervention, for this, only the number of safe observations was taken for the purpose of To know the effect of the positive reinforcement feedback that occurs in the observation of safe behaviors in the study period. It was found that there is a significant difference in safe behaviors after performing the feedback for a confidence level of $95 \%$ and a reduction of $85.7 \%$ of disabling accidents in the study period.

Keywords: Safety Program Based on Behavior; Leadership and Accident Reduction. de su experiencia puede poner esa misma situación de riesgo en un contexto menos favorable para afirmar que aumentaría el riesgo de ocurrencia de accidente y que no es suficiente con informar los actos y condiciones inseguras sino gestionarlos para corregirlos y levantarlos, ésta es la tarea del facilitador y es la característica de un buen líder. Lograr el entendimiento de este concepto por parte de los líderes o supervisores es lo que hace la diferencia; así, el cambio de la conducta hacia la seguridad es lo que permitirá fortalecer la cultura de prevención. Con el involucramiento de la supervisión en la aplicación de un Programa de Seguridad Basado en el Comportamiento (PSBC), Martínez, C. (2014) explica que en empresas del rubro de manufactura, menciona que se logró reducir la frecuencia de accidentes con incapacidad laboral en un $60.8 \%$ y también una disminución del $44.4 \%$ del total de casos de accidentes con y sin incapacidad laboral. Por el lado de los trabajadores está la resistencia al cambio de sus comportamientos inseguros que se debe a la presencia de los paradigmas que se presentan en el colectivo, y es aquí donde se recuerda a Espluga (2004) cuando menciona que "hay que tener en cuenta que las personas no cambian voluntariamente de actitudes, ya que uno no puede decidir cambiar una cosa de la que no es consciente...tiene que ser un observador quien juzgue tal cosa". p. 2.
Jasiulewicz-Kaczmarek, M., Szwedzka, K. y Szczuka, M. (2015) explican que una herramienta que puede ayudar a las empresas a seguir la evolución de su cultura de seguridad es la curva de Bradley de DuPont que ilustra el espectro de una cultura de seguridad a medida que madura pasando de una etapa reactiva a proactiva. El modelo se caracteriza por estar conformada por cuatro etapas, a saber:

1. Etapa reactiva: cuando las empresas manejan sus problemas de seguridad por instinto natural, centrándose en el cumplimiento de procedimientos en lugar de una fortalecer la cultura de seguridad. La responsabilidad se delega al Gerente de Seguridad, y generalmente hay una falta de compromiso de la administración con los problemas de seguridad.

2. Etapa dependiente: existe cierto compromiso de gestión, los supervisores generalmente son responsables del control de seguridad, el énfasis y los objetivos. La atención a la seguridad se convierte en una condición de empleo, pero con énfasis en el miedo y la disciplina, las reglas y los procedimientos. Tales compañías valoran a toda su gente y proporcionarán seguridad y formación.

3. Etapa independiente: estas empresas se centra en el conocimiento personal de los problemas y métodos de seguridad, así como el compromiso y los estándares. La administración de seguridad se internaliza y acentúa el valor personal y la atención del individuo. Estas compañías participan en prácticas y hábitos de seguridad activa y reconocen los logros personales en seguridad

4. Etapa interdependiente: cuando los trabajadores se ayudan activamente unos a otros a ajustarse a las iniciativas de seguridad: en cierto sentido, se convierten en "guardianes de otros". Contribuyen a armar una red de seguridad y tienen un fuerte sentido de orgullo organizacional en sus esfuerzos por la seguridad.

Con la implementación de un Programa de Seguridad Basado en el Comportamiento (PSBC) se quiere acercar a la fase Interdependiente de la curva de Bradley en dónde los trabajadores tienen un sentimiento de propiedad y creen que trabajando en equipo se puede alcanzar el objetivo de cero accidentes.

EI PSBC no reemplaza ni elimina a los componentes de la gestión de seguridad, sino que se integra a las estrategias del sistema de gestión de seguridad, el objetivo es potenciar a los trabajadores en la prevención de accidentes, aplicando métodos basados en el comportamiento humano de manera que se hace práctico, efectivo y porque garantiza resultados satisfactorios (Montero, 2003). EI PSBC 
describe la forma de prevenir accidentes, lesiones y pérdidas materiales en el lugar de trabajo, identifica los problemas de seguridad permitiendo evaluar las áreas problemáticas y crear pautas de seguridad basadas en el comportamiento dado que el cambio de comportamiento no se produce cambiando a la persona, sino cambiando la percepción de los riesgos del lugar de trabajo. Implica observar el comportamiento y detectarlo como "en riesgo" o inseguro, seguido de dirigir o modificar el comportamiento para lograr la tarea segura, busca transferir el control del incidente a las manos del empleado, así el empleado se vuelve proactivo hacia su propia seguridad y no es una víctima de su entorno. La técnica aplicada es la observación y la retroalimentación entre pares, donde se registran las observaciones en una lista de control de comportamientos, y se entrega retroalimentación al observado. La retroalimentación con refuerzo positivo puede darse en público o en privado (formativa), esta última se da en privado ya que puede percibirse como castigo. (Jasiulewicz-Kaczmereck, M. et. al. (2015). En la gestión del PSBC, se quiere encontrar cambios positivos para la seguridad y la reducción de accidentes en un proceso de mejora continua desde la identificación de los comportamientos inseguros durante la realización de la observación de la tarea en el lugar de trabajo, registrando en la cartilla de observaciones y devolviendo retroalimentación a los observados, con la finalidad de fortalecer la cultura de seguridad (Martínez, C. 2015)

Los comportamientos críticos identificados en las tarjetas de observadores son el resultado de la aplicación del proceso denominado DOIT. Meliá (2007) explica que este proceso se inicia definiendo (D) cuales son los comportamientos críticos de manera objetiva, los mismos que provienen de un histórico de comportamientos observados que se quieren evitar. Estos comportamientos definidos son (O) observados en un periodo de tiempo para conocer la frecuencia con la que se presentan, para cada observación le sigue una intervención (I), éstas son de retroalimentación con refuerzo positivo con la escala de medición nominal, las observaciones se realizan con una frecuencia determinada. Realizando las intervenciones de manera permanente se pueden testear $(T)$ los resultados del programa, éste control mide la efectividad del programa diseñado, en donde la variable independiente son las intervenciones dadas en el proceso y la variable dependiente las Observaciones Seguras, también se pueden considerar otras como la Frecuencia de accidentes, Severidad de accidentes, Costos de pérdidas, etc.
Geller (2005) explica que para aplicar el proceso DOIT es importante mejorar las condiciones de trabajo para facilitar las intervenciones, dice "intervención significa cambiar las condiciones externas del sistema para hacer que el comportamiento seguro sea más probable que el comportamiento de riesgo".p.542.

Montero (1999) explica que el término Porcentaje de Observaciones Seguras se emplea porque define el mensaje que se le quiere enviar a los trabajadores, "no representa una medida de la seguridad, sino solamente el porcentaje de prácticas claves consideradas seguras o realizadas correctamente al ser observadas". p. 89. Este resultado se presenta para conocimiento de los trabajadores con el propósito de incentivar a mantener el éxito del rendimiento de seguridad. Sobre el resultado recogido después de realizar la intervención debe entenderse que

“...se establece en términos de logro y es fácil obtener un alto porcentaje, esto pone todo proceso en un modo de logro en lugar de la típica falla o pérdida métrica utilizada para medir el rendimiento de seguridad (por ejemplo, número de lesiones o incidentes de daños a la propiedad y costos de compensación para trabajadores". (Geller, 2005, p. 549).

Esto es debido a la preocupación de B.F. Skinner sobre los sentimientos y actitudes de las personas que se reflejan en su antipatía hacia el uso del castigo (consecuencias negativas) para motivar el cambio de comportamiento. "Skinner hace alusión a que se debe liberar al hombre no de todo el control, sino de ciertos tipos de control." (Skinner, 1971, p. 41)". Citado por Geller (2005). p. 542.

En la presente investigación se recogen los resultados de las observaciones seguras para evaluar el efecto de las intervenciones, así Geller (2005) explica que "los comportamientos pueden observarse y medirse objetivamente antes y después de que se inicia un proceso de intervención donde se proporciona la retroalimentación para cultivar la mejora". p. 542.

Martín (2014) menciona que el Dr. Albert Ellis fue el creador de la Terapia Racional Emotiva Conductual (TREC) con el propósito de identificar en las personas sus pensamientos irracionales y ayudar a reemplazarlos por pensamientos racionales o eficientes, a fin de lograr sus metas personales. Esta teoría se basa en el modelo Activator - Behavior Consequence (Modelo $\mathrm{ABC}$ ) que fue publicado en 
la década de los años 60 , explicando que las personas responden no tanto a la situación externa sino a la interpretación que hacen de una situación. En la presente investigación se aplicó este modelo para reforzar los comportamientos seguros a partir de sus antecedentes (activadores) o de sus consecuencias.

Alfaro (2018) afirma que "bajo este modelo deberán establecerse aquellos factores (Activadores o antecedentes) que preceden y facilitan la ocurrencia de la conducta identificada como preocupante y también aquellos eventos que fortalecen su mantención en el tiempo (Consecuencias). Este análisis permitirá facilitar los planes de acción asociados a la identificación de conductas". (p.16)

Garlapati, Siddiqui y Al-Shatti (2013) explican que "el modelo $A B C$ que es útil para comprender el por qué algunos trabajadores siguen realizando hábitos de riesgo". p. 485. Así, identificando los antecedentes (eventos previos al comportamiento) y consecuencias (eventos posteriores al comportamiento) que pueden estar influyendo en la ejecución de un determinado comportamiento, para modificarlos de forma que influyan positivamente en el comportamiento. (Montero, 2010)

El problema en la presente investigación se identifica cuando no se entienden los principios de la teoría de la seguridad basada en el comportamiento; así, el cambio de los comportamientos hacia la seguridad se consigue realizando intervenciones eficientes cada vez que se realizan las observaciones de comportamientos y no priorizando sólo el cumplimiento de un número de observaciones a realizar como meta del mes, esta situación genera que los trabajadores cumplan con la seguridad mientras se les está observando; consecuentemente, los observadores dejan de creer en el programa de seguridad que están utilizando. Por ello, el objetivo de la presente investigación es determinar si existe una la relación significativa entre las observaciones antes y después de aplicar la retroalimentación con refuerzo positivo, para ello se plantearon las siguientes hipótesis $(\mathrm{Hp})$ : Existirá una diferencia significativa entre las observaciones de comportamiento seguro antes (pre-test) que el observador realice la retroalimentación con refuerzo positivo y la observación después de dicha intervención (post-test) y (He): El cambio hacia los comportamientos seguros contribuirá a mejorar los resultados de seguridad.

Esta investigación se justifica dado que la implementación de la SBC está contemplada en la Ley $\mathrm{N}^{\circ}$ 29783, Ley de seguridad y salud en el trabajo, en el Título IV, Capítulo I, Artículo 18, inciso e): "Fomentar la cultura de la prevención de riesgos laborales para que toda la organización interiorice los conceptos de la prevención y proactividad, promoviendo comportamientos seguros".

Por lo expuesto, el tema de la investigación es trascendente, porque contribuye a entender cómo funciona la SBC para prevenir accidentes laborales desde el diseño de la Tarjeta de observadores y realizar las intervenciones eficientemente para reforzar los comportamientos seguros y hacer notar los comportamientos inseguros a través de sus consecuencias, la presentación de resultados y el efecto en la reducción de la frecuencia de accidentes, que en el presente caso se aplicó en una planta de producción de bebidas gaseosas de Lima (Perú).

\section{METODOLOGÍA:}

\section{Tipo y diseño de la investigación}

La presente investigación es aplicada, en razón a que se utilizó la teoría de la SBC en las tareas propias del personal de producción.

El método que se utilizó en la investigación fue el deductivo, se analizaron los resultados de las observaciones de comportamientos seguros registrados en las Tarjetas de Observación y los resultados del indicador de frecuencia de accidentes entre los años 2010 y 2015, resaltando el efecto de la implementación de la SBC.

El diseño de la investigación es pre experimental de pre prueba y post prueba con un solo grupo, considera dos mediciones de la variable dependiente antes y después de la presencia de la variable independiente.

\section{Población de estudio}

La población en estudio estuvo conformada por los resultados de las Tarjetas de Observadores y los reportes de accidentes con incapacidad laboral entre los años 2010 y 2015.

\section{Selección de la muestra}

Se trabajó con 26 observadores (supervisores) de diferentes áreas que aplicaron sus tarjetas de observación de manera semanal y estaban distribuidos de la siguiente manera: producción (9), mantenimiento (4), almacén de productos y envases (7), logística (1), aseguramiento de calidad (1), procesos (3) y capital humano (1). La selección de muestra fue probabilística, considerando el total de observadores $(n<30)$; para fines del análisis se 
definió el diseño de muestreo seleccionando aleatoriamente a 12 observadores al mes durante el periodo de la investigación.

\section{Tamaño de la muestra}

El tamaño de muestra lo determinó el número de comportamientos identificados como seguros en las Tarjetas de Observación aplicadas por los observadores seleccionados, en el periodo de agosto del 2013 a diciembre del 2015, es decir que se trabajó con una base de datos de 29 resultados. Para encontrar si hay diferencia significativa entre ellas se han apareado mes a mes, para ello se ha tomado un primer mes como pre-test y el subsiguiente como post-test.

\section{Técnicas de recolección de datos.}

La técnica de recolección de datos fue la revisión de los Reportes mensuales de las observaciones de comportamientos seguros, de donde se obtuvo la información para la presente investigación y los Reportes mensuales de la frecuencia de accidentes con incapacidad laboral.

\section{Análisis e interpretación de la información}

Los resultados de las Tarjetas de observadores constituyen la variable dependiente, las mismas que son de naturaleza nominal. Hernández, Fernández y Baptista (2014) definen el emparejamiento o la técnica de apareo, que consiste en igualar a los grupos en relación con alguna variable específica que puede influir de modo decisivo en la o las variables dependientes. La variable independiente o tratamiento es la retroalimentación con refuerzo positivo, la escala de medición es nominal en donde la categoría observada no tiene orden ni jerarquía; lo que se mide se coloca en una u otra categoría, lo cual indica tan sólo diferencias respecto de una categorías, ninguna de las categorías implica mayor jerarquía que la otra, sólo reflejan diferencias en la variable.

Respecto a la prueba de McNemar aplicada en el análisis de datos para muestras relacionadas, Pértega y Pita (2004) explican que el interés se centra en comparar si las mediciones efectuadas en dos momentos diferentes (normalmente antes y después de alguna intervención) son iguales o si, por el contrario, se produce algún cambio significativo.

Con la aplicación de las Tarjetas de Observación se han identificado comportamientos seguros; por lo que, el estadístico inferencial que demuestra el efecto que tiene la retroalimentación con refuerzo positivo (variable independiente) sobre el comportamiento de los trabajadores, es la prueba no paramétrica de McNemar para aceptar o rechazar la hipótesis planteada (ver Tabla1).

En la Tabla 2, se presenta la Matriz de análisis de datos para las variables presentadas.

Tabla 1. Matriz de identificación de variables.

\begin{tabular}{|l|l|l|l|l|}
\hline \multicolumn{1}{|c|}{ Hipótesis } & \multicolumn{1}{|c|}{$\begin{array}{c}\text { Variable } \\
\text { Independiente (VI) }\end{array}$} & \multicolumn{1}{|c|}{ Indicador (VI) } & \multicolumn{1}{c|}{$\begin{array}{c}\text { Variable } \\
\text { Dependiente (VD) }\end{array}$} & \multicolumn{1}{|c|}{ Indicador (VD) } \\
\hline $\begin{array}{l}\text { Hp: Existirá una diferencia significativa } \\
\text { entre las observaciones de comportamiento } \\
\text { seguro antes (pre-test) que el observador } \\
\text { realice la retroalimentación con refuerzo } \\
\text { positivo y la observación después de dicha } \\
\text { intervención (post-test). }\end{array}$ & $\begin{array}{l}\text { La retroalimentación } \\
\text { con refuerzo positivo } \\
\text { aplicados al perso- } \\
\text { nal de producción. }\end{array}$ & $\begin{array}{l}\text { Número de Tarjetas } \\
\text { de observación de } \\
\text { comportamientos } \\
\text { seguros al mes. }\end{array}$ & $\begin{array}{l}\text { Los resultados de } \\
\text { los comportamientos } \\
\text { seguros registrados. }\end{array}$ & $\begin{array}{l}\text { Y1: Número de ob- } \\
\text { servaciones seguras } \\
\text { registradas por mes. }\end{array}$ \\
\hline $\begin{array}{l}\text { He: El cambio hacia los comportamientos } \\
\text { seguros contribuirá a reducir la frecuencia } \\
\text { de accidentes con incapacidad laboral. }\end{array}$ & $\begin{array}{l}\text { La retroalimentación } \\
\text { con refuerzo positivo } \\
\text { aplicados al perso- } \\
\text { nal de producción }\end{array}$ & $\begin{array}{l}\text { Número de Tarjetas } \\
\text { de observación de } \\
\text { comportamientos } \\
\text { seguros al mes. }\end{array}$ & $\begin{array}{l}\text { Número de acciden- } \\
\text { tes ocurridos al mes. }\end{array}$ & $\begin{array}{l}\text { Y2: Frecuencia de } \\
\text { accidentes }\end{array}$ \\
\hline
\end{tabular}

Fuente: Elaboración propia

Tabla 2. Matriz de análisis de datos.

\begin{tabular}{|l|l|l|l|l|}
\hline \multicolumn{1}{|c|}{ Variables } & \multicolumn{1}{|c|}{ Indicador } & $\begin{array}{l}\text { Escala de } \\
\text { medición }\end{array}$ & \multicolumn{1}{|c|}{$\begin{array}{l}\text { Estadísticos } \\
\text { Descriptivos }\end{array}$} & \multicolumn{1}{c|}{ Análisis Inferencial } \\
\hline $\begin{array}{l}\text { Resultados de la Observación } \\
\text { de comportamientos seguros. }\end{array}$ & $\begin{array}{l}\text { Número de observaciones } \\
\text { seguras registradas por mes. }\end{array}$ & $\begin{array}{l}\text { Nominal } \\
\text { dicotómica. }\end{array}$ & $\begin{array}{l}\text { Conteo } \\
\text { Frecuencia }\end{array}$ & $\begin{array}{l}\text { Prueba No paramétrica de Mc- } \\
\text { Nemar. }\end{array}$ \\
\hline $\begin{array}{l}\text { Desempeño del indicador de } \\
\text { seguridad. }\end{array}$ & Frecuencia de Accidentes. & Razón & Frecuencia. & $\begin{array}{l}\text { Prueba paramétrica T de Student } \\
\text { para muestras relacionadas. }\end{array}$ \\
\hline
\end{tabular}

Fuente: Elaboración propia 
Para el caso de la Frecuencia de accidentes con incapacidad laboral, en el análisis inferencial se aplicó la prueba paramétrica $\mathrm{T}$ de Student para muestras relacionadas para aceptar o rechazar la hipótesis planteada (ver Tabla 1). El nivel de confianza aplicado fue de $95 \%$.

\section{RESULTADOS}

En la presente investigación se siguió el diagrama de flujo que se presenta en la Figura 1, a fin de que los resultados obtenidos aseguren el éxito del programa, este es el aporte de la investigación. Se aplicó el método científico DOIT y el modelo $A B C$ en el PSBC. Así, en la Figura 1 se presenta el diagrama de flujo que muestran los actores que intervienen y sus responsabilidades a fin de lograr el cambio de los comportamientos hacia la seguridad y consecuentemente la reducción de los accidentes laborales. Se inicia con el compromiso de la Alta dirección para aplicar la teoría de la Seguridad Basada en los Comportamientos plasmada en el PSBC aplicado, el mismo que forma parte del sistema de seguridad de la planta. Se destacan los roles del Comité de Seguridad como ente coordinador responsable del programa, los Líderes de los equipos de trabajo (conformado por personal de las diferentes áreas o líneas de producción de la planta) quienes tienen la responsabilidad de realizar las observaciones, la retroalimentación con refuerzo positivo, resaltar los comportamientos preocupantes e identificar las condiciones inseguras, verificar el levantamiento de las mismas y evaluar si hubo mejora de los comportamientos observados a fin de lograr que el personal a su cargo esté siempre motivado a trabajar seguro, a fin de reforzar su propia cultura de seguridad. El responsable de seguridad quien recepciona las tarjetas de los observadores y lleva la estadística para encontrar los aquellos comportamientos preocupantes que se repiten (desvíos recurrentes) a fin de que se discutan en el Comité de seguridad y planteen las medidas correctivas necesarias. El dueño del proceso es el responsable de implementar las medidas correctivas a través de las Charlas de seguridad o gestionar las necesidades identificadas a través de la compra e instalación o solicitarlas a mantenimiento de planta. La Tarjeta de Observadores aplicada fue diseñada para observar comportamientos definidos en todas las áreas de la planta, observando tanto a personal propio o de terceros. En ella se definen 05 categorías de comportamientos objetivos: Reacción del personal, Exposición de la gente, Equipos de protección personal, Actividades generales y Operaciones con vehículos. Se definió la calificación de los comportamientos como seguro, preocupante (inseguro) o No Aplica (NA). En la parte final se calcula el resultado de las observaciones como Tasa de comportamiento seguro, es decir el Porcentaje de observaciones seguras encontradas.

En la Figura 2 se muestran los Resultados del Porcentaje de Observaciones Seguras por cada categoría de comportamiento objetivo correspondiente al mes de agosto del 2014. En este resultado del mes se observan los logros expresados en porcentaje para cada una de las cinco (05) categorías de comportamientos objetivos. Estos resultados se publican en la planta para conocimiento de los trabajadores.

En la Figura 3 se muestran los resultados de la Frecuencia mensual de accidentes con incapacidad laboral en el periodo enero 2010 a diciembre del 2015 y los resultados de las observaciones de comportamiento seguro que se dieron desde la implementación de la SBC en el mes de agosto 2013. Se observa que entre Enero-2010 a Agosto-2013, periodo previo a la implantación de la SBC, se identifica al mes de enero del 2012 como el más crítico en la frecuencia de accidentes con 7 casos, luego de la implementación de la SBC hay una marcada reducción de accidentes acompañada de resultados crecientes de observaciones seguras. El promedio de accidentes con incapacidad laboral después de la implementación se reduce en un $77 \%$ (de 2.53 a 0.58 casos al mes). El periodo de implementación de la SBC no fue suficiente la para conseguir la meta de cero accidentes, los resultados demuestran que se fortaleció su propia cultura de seguridad con la participación de los líderes y trabajadores en temas de seguridad.

En la Tabla 3 se muestra un ejemplo de aplicación para un comportamiento específico: Uso de guantes anticorte, en donde se muestra un listado de antecedentes para el comportamiento mencionado dando lugar a una de las consecuencias. Para aumentar un determinado comportamiento, en la retroalimentación se puede actuar sobre los antecedentes o sobre las consecuencias.

En la Figura 4 se presenta la Frecuencia anual de accidentes ocurridos en el periodo 2010 y 2015, se observa que el promedio de accidentes con incapacidad laboral entre los años 2010 al 2013 fue de 32 casos al año, mientras que para los años 2014 y 2015 , el promedio se redujo a 4 casos al año, ello significa una reducción del $87.5 \%$. Ello se debe a la implantación de la SBC, a partir del mes de agosto del 2013. El promedio de accidentes entre los años 2010 al 2012 fue de 35 casos al año, al tomar este periodo como base para comparar los 


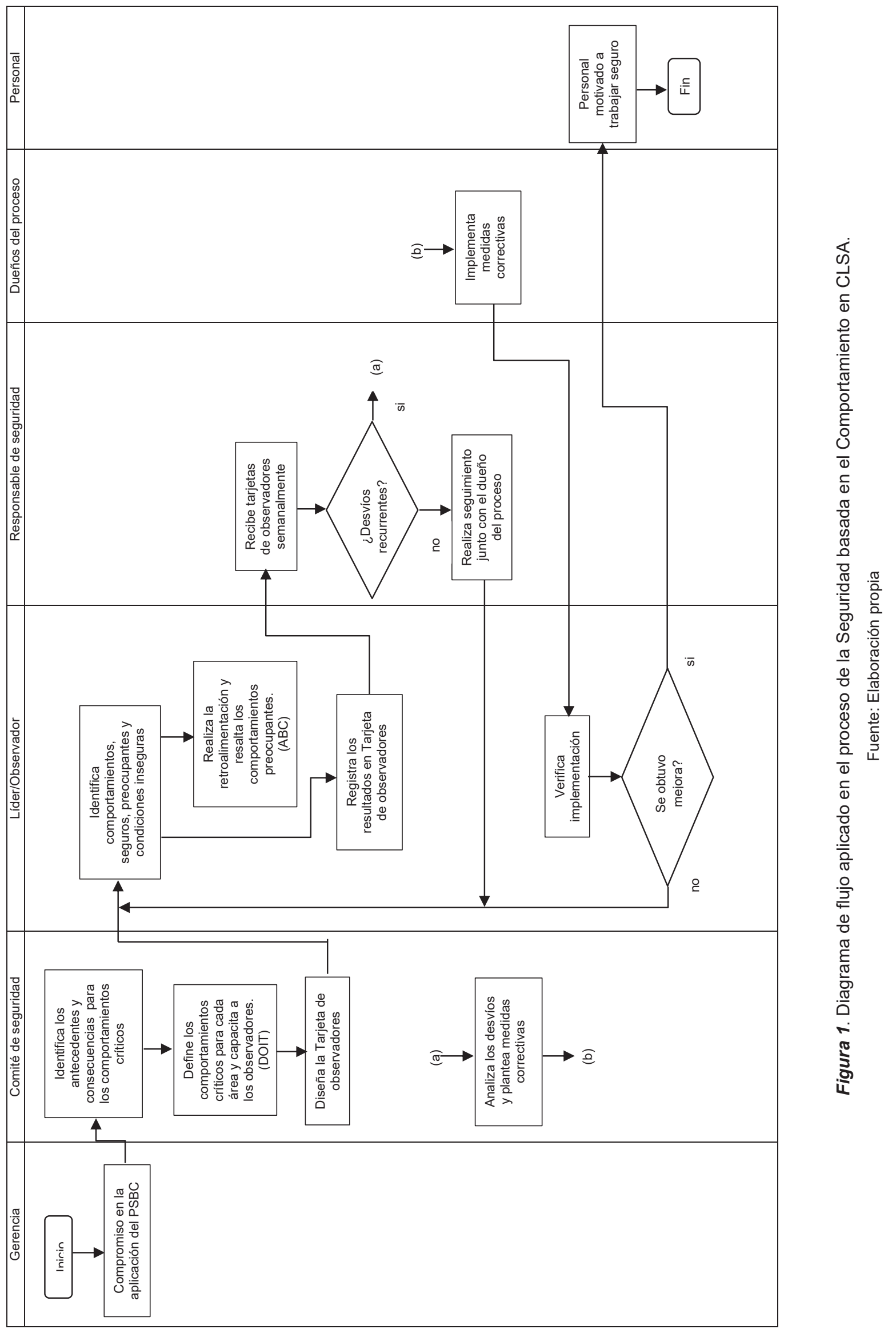




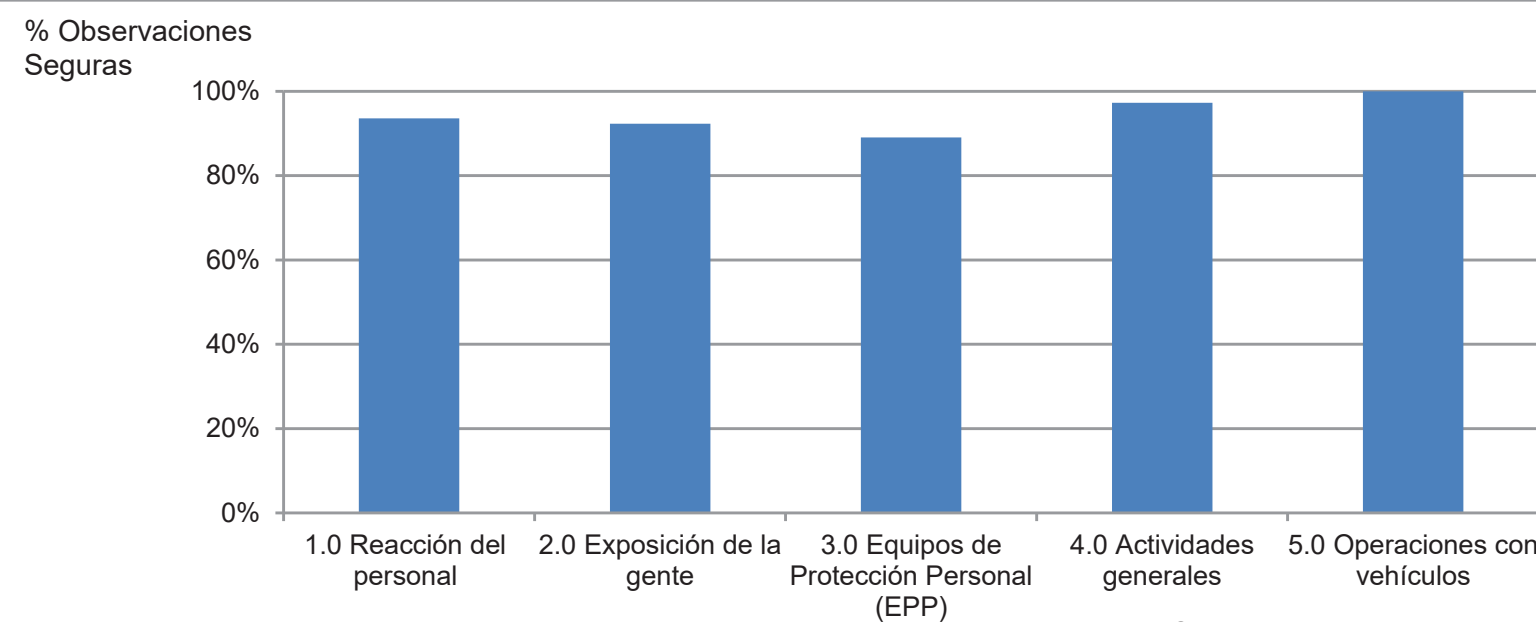

Comportamientos

Figura 2. Resultados de Comportamiento Seguro del área de Producción, Agosto 2014

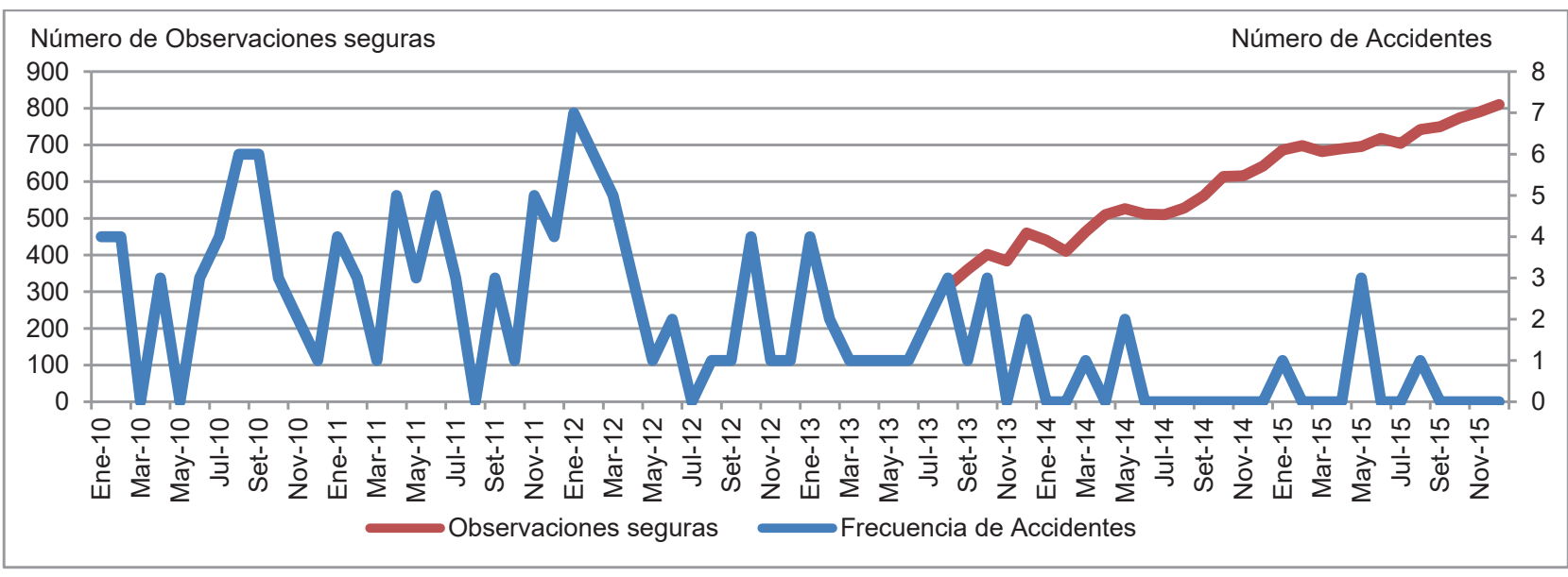

Figura 3. Comparación mensual entre la frecuencia de accidentes y las observaciones de comportamiento seguros en el periodo 2010 al 2015.

Fuente: Elaboración propia

Tabla 3. Ejemplo de aplicación del Modelo ABC para un comportamiento específico.

\begin{tabular}{|l|l|}
\hline Comportamiento: No usar guantes anticorte & \\
\hline Antecedentes: & Consecuencias: \\
No querer usar guantes anticorte & Puedo operar el panel de control \\
Creer que no puede accidentarse & Puedo terminar accidentado \\
Los deja en otro sitio & Pueden llamarme la atención \\
Pienso que a nadie le interesa & Puedo ser suspendido \\
Veo que otros no los usan & No me preocupan los demás \\
No recuerdo un accidente reciente & Sentir menos molestias \\
& Se ensucian fácilmente \\
\hline
\end{tabular}

Fuente: Elaboración propia 


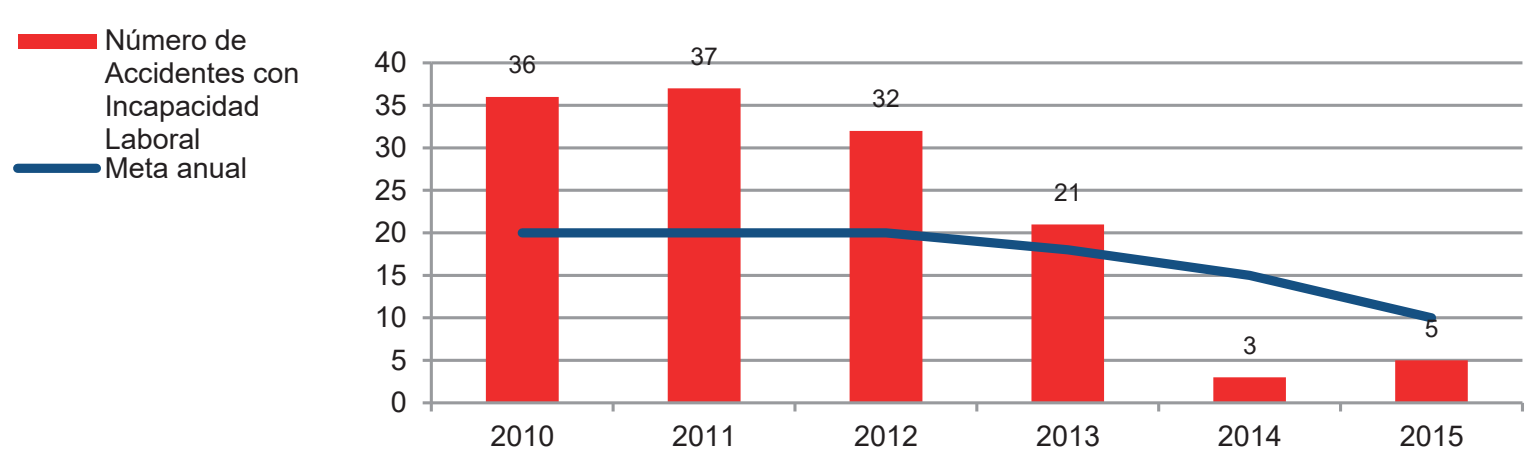

Figura 4. Frecuencia anual de accidentes en el periodo 2010 al 2015.

Fuente: Elaboración propia

siguientes años encontramos que para el año 2013 que cerró con 21 accidentes, se logró reducir en $40 \%$ (de 35 a 21 casos), en ese mismo orden para el año 2014 que cerró con 3 accidentes, se logró reducir en $91.4 \%$ (de 35 a 3 casos) y al comparar con el año 2015 que cerró con 5 accidentes, se logró reducir en $85.7 \%$.

\subsection{Comprobación de las hipótesis.}

En la Tabla 1 se presenta la hipótesis principal $(\mathrm{Hp})$ de la investigación: Existirá una diferencia significativa entre las observaciones de comportamiento seguro antes (pre-test) que el observador realice la retroalimentación con refuerzo positivo y la observación después de dicha intervención (post-test), es por ello que en la Tabla 4 se muestran los resultados de comportamientos seguros en el periodo de agosto 2013 a diciembre 2015, en donde la columna OS, representa la sumatoria de las observaciones seguras del mes, con la finalidad de comparar el resultado con el siguiente mes es que se ha agregado la columna OS2. En la columna "dif" (diferencia) se ha colocado la diferencia entre OS1 y OS2.

De la Fuente (2016) explica que al aplicar la prueba de McNemar en la Tabla de Contingencia se asignan las diferencias de los valores identificados como "b" y "c"; sí el resultado de la diferencia es positiva, se le asignará el valor de 1 en la columna de "b" y si la diferencia es negativa se asignará el valor de 1 en la columna "c". Para los resultados obtenidos se encuentran diferencias positivas y negativas; sólo se contarán con los resultados de las columnas "b" y "c, también se muestra que el total de diferencias positivas es 21 y de las negativas es 8 . La suma de $b+c$ es el número de casos que cambiaron, considerando la hipótesis nula, se espera de $(b+c) / 2$ casos cambien en una dirección y $(b+c) / 2$ casos cambien en otra dirección.

Para contrastar la hipótesis principal, se plantean las Hipótesis nula $(\mathrm{Ho})$ y la Hipótesis alterna $\left(\mathrm{H}_{1}\right)$

$\mathrm{H}_{0}$ : La retroalimentación con refuerzo positivo no induce cambios significativos en los comportamientos seguros.

$\mathrm{H}_{1}$ : La retroalimentación con refuerzo positivo induce cambios significativos en los comportamientos seguros.

El estadístico corregido para $(b+c) \geq 20$ es:

$X^{2}$ McNemar $=(\mid \mathrm{b}-\mathrm{cl}-1)^{2} /(\mathrm{b}+\mathrm{c})$, se acepta $\mathrm{H}_{0}$ si $X^{2} \operatorname{McNemar}<X^{2}(\alpha / 2,1)$

Reemplazando valores se encuentra que $\mathrm{X}^{2} \mathrm{McNe}$ mar $=4.9655$ y que $X^{2}(0.05,1)=3.8415$, por lo que se rechaza la Hipótesis nula y se acepta la Hipótesis del investigador, es decir que las intervenciones con refuerzo positivo afectan el comportamiento seguro del personal de producción.

En la Tabla 1, se presenta la hipótesis específica $(\mathrm{He})$ de la investigación: El cambio hacia los comportamientos seguros contribuirá a reducir la frecuencia de accidentes, es por ello que para la evaluación de la variable dependiente Frecuencia de accidentes presentados en la Figura 4, se aplicó la prueba T de Student para muestras relacionadas.

Para contrastar la hipótesis específica se plantean las Hipótesis nula $(\mathrm{Ho})$ y la Hipótesis alterna $\left(\mathrm{H}_{1}\right)$ 
$\mathrm{H}_{0}$ : No hay diferencia significativa entre las medias de la frecuencia de accidentes con incapacidad laboral antes y después de cada retroalimentación con refuerzo positivo.

$\mathrm{H}_{1}$ : Hay diferencia significativa entre las medias de la frecuencia de accidentes con incapacidad laboral antes y después de cada retroalimentación con refuerzo positivo.

En la Tabla 5 se muestra el resultado de la prueba $T$ de Student, donde la significancia encontrada es 0.175 es mayor que $\alpha=0.05$, se acepta la hipótesis nula, por lo que el periodo de estudio no fue suficiente para lograr la meta de cero accidentes.

\section{DISCUSIÓN}

1. Con la finalidad de demostrar la hipótesis se siguieron los pasos indicados en el diagrama de flujo de la Figura 1. Se aseguró que el diseño de las tarjetas de observadores refleje la necesidad de levantar aquellos comportamientos identificados como críticos de la planta, para ello se utiliza el procedimiento

Tabla 4. Resultados de Comportamientos Seguros - Agosto 2013 a diciembre 2015.

\begin{tabular}{|c|c|c|c|c|c|c|c|c|c|c|}
\hline Mes & Reaccseg & Exposeg & EPPseg & Actseg & Vehseg & os1 & os2 & dif. & B & C \\
\hline ago-13 & 54 & 92 & 140 & 24 & 4 & 314 & 360 & 46 & 1 & 0 \\
\hline set13 & 46 & 88 & 178 & 34 & 14 & 360 & 402 & 42 & 1 & 0 \\
\hline oct-13 & 42 & 112 & 186 & 44 & 18 & 402 & 384 & -18 & 0 & 1 \\
\hline nov-13 & 42 & 112 & 174 & 40 & 16 & 384 & 460 & 76 & 1 & 0 \\
\hline dic-13 & 68 & 132 & 198 & 46 & 16 & 460 & 440 & -20 & 0 & 1 \\
\hline ene-14 & 64 & 150 & 162 & 44 & 20 & 440 & 410 & -30 & 0 & 1 \\
\hline feb-14 & 60 & 132 & 170 & 44 & 4 & 410 & 464 & 54 & 1 & 0 \\
\hline mar-14 & 84 & 142 & 186 & 44 & 8 & 464 & 510 & 46 & 1 & 0 \\
\hline$a b r-14$ & 90 & 174 & 174 & 60 & 12 & 510 & 526 & 16 & 1 & 0 \\
\hline may-14 & 84 & 168 & 184 & 82 & 8 & 526 & 512 & -14 & 0 & 1 \\
\hline jun-14 & 94 & 154 & 170 & 80 & 14 & 512 & 510 & -2 & 0 & 1 \\
\hline jul-14 & 84 & 152 & 200 & 66 & 8 & 510 & 528 & 18 & 1 & 0 \\
\hline ago-14 & 88 & 168 & 196 & 72 & 4 & 528 & 562 & 34 & 1 & 0 \\
\hline set14 & 80 & 202 & 184 & 88 & 8 & 562 & 614 & 52 & 1 & 0 \\
\hline oct-14 & 92 & 206 & 202 & 98 & 16 & 614 & 616 & 2 & 1 & 0 \\
\hline nov-14 & 98 & 212 & 200 & 94 & 12 & 616 & 642 & 26 & 1 & 0 \\
\hline dic-14 & 98 & 244 & 196 & 96 & 8 & 642 & 686 & 44 & 1 & 0 \\
\hline ene-15 & 118 & 224 & 228 & 98 & 18 & 686 & 698 & 12 & 1 & 0 \\
\hline feb-15 & 114 & 252 & 216 & 100 & 16 & 698 & 682 & -16 & 0 & 1 \\
\hline mar-15 & 116 & 232 & 226 & 92 & 16 & 682 & 690 & 8 & 1 & 0 \\
\hline abr-15 & 118 & 230 & 210 & 116 & 16 & 690 & 696 & 6 & 1 & 0 \\
\hline may-15 & 126 & 252 & 206 & 98 & 14 & 696 & 718 & 22 & 1 & 0 \\
\hline jun-15 & 126 & 254 & 216 & 108 & 14 & 718 & 704 & -14 & 0 & 1 \\
\hline jul-15 & 122 & 258 & 198 & 120 & 6 & 704 & 742 & 38 & 1 & 0 \\
\hline ago-15 & 124 & 244 & 232 & 126 & 16 & 742 & 750 & 8 & 1 & 0 \\
\hline set15 & 120 & 278 & 226 & 114 & 12 & 750 & 774 & 24 & 1 & 0 \\
\hline oct-15 & 126 & 268 & 246 & 120 & 14 & 774 & 790 & 16 & 1 & 0 \\
\hline nov-15 & 126 & 274 & 224 & 164 & 2 & 790 & 810 & 20 & 1 & 0 \\
\hline dic-15 & 118 & 286 & 248 & 132 & 26 & 810 & 802 & -8 & 0 & 1 \\
\hline Totales & & & & & & & & & 21 & 8 \\
\hline
\end{tabular}

Fuente: Elaboración propia 
Tabla 5. Prueba T de Student para muestras relacionadas.

\begin{tabular}{|c|c|c|c|c|c|c|c|c|c|}
\hline & & \multicolumn{5}{|c|}{ Diferencias relacionadas } & \multirow{3}{*}{$\mathbf{t}$} & \multirow{3}{*}{ GI } & \multirow{3}{*}{$\begin{array}{c}\text { Sig. } \\
\text { (bilateral) }\end{array}$} \\
\hline & & \multirow[t]{2}{*}{ Media } & \multirow[t]{2}{*}{ Desviación típ. } & \multirow{2}{*}{$\begin{array}{c}\text { Error típ. de la } \\
\text { media }\end{array}$} & \multicolumn{2}{|c|}{$\begin{array}{c}95 \% \text { Intervalo de confianza } \\
\text { para la diferencia }\end{array}$} & & & \\
\hline & & & & & Inferior & Superior & & & \\
\hline Par 1 & Antes - Después & 6.200 & 8.408 & 3.760 & -4.240 & 16.640 & 1.649 & 4 & 0.175 \\
\hline
\end{tabular}

Fuente: Elaboración propia

DOIT, dado a conocer por Geller (2005), aquí es importante considerar que estos comportamientos identificados corresponden a una fotografía de un momento, por lo que pueden cambiar con el tiempo; es necesario volver a analizar para actualizar los comportamientos a observar.

2. En la Tabla 3 se presenta un ejemplo de aplicación del modelo ABC para hacer una eficiente retroalimentación con refuerzo positivo, este modelo tiene como principio entender por qué las personas responden no tanto a la situación externa sino a la interpretación que hacen de una situación, por ello Garlapati, et. al. (2013) explica que sirve para entender por qué algunos trabajadores siguen realizando actos inseguros; en la retroalimentación se refuerzan de manera positiva los comportamientos seguros para que se mantengan en el tiempo y en el caso de comportamientos preocupantes se refuerzan las consecuencias a las que se expone, se refuerza la conducta y no a la persona, a fin de que interprete una situación de peligro que no advirtió y que puede causarle daño a su salud.

3. Los Resultados de Comportamientos Seguros (ver Figura 2), miden el porcentaje de Observaciones Seguras para las categorías de comportamientos objetivos, de acuerdo con Montero (1999) y Geller (2005), explican que es el logro alcanzado de las prácticas claves consideradas como seguras y que tienen valores próximos a $100 \%$; se ven como metas de seguridad fácilmente alcanzables.

4. En la comparación mensual entre la frecuencia de accidentes y las observaciones de comportamiento seguro en el periodo de estudio (ver Figura 3) y en el comportamiento de la frecuencia anual de accidentes en el periodo de estudio (ver Figura 4), se observa en ambas figuras una reducción en la ocurrencia de accidentes con incapacidad laboral a partir del mes que se inició el PSBC, lo que corrobora lo mencionado por Montero (2003) cuando responde a la pregunta ¿Por qué tanta atención en la denominada Seguridad Basada en los Comportamientos?, la respuesta es porque garantiza resultados satisfactorios.

5. Se ha encontrado que el efecto de las intervenciones de retroalimentación con refuerzo positivo producen cambios significativos en los comportamientos de los trabajadores hacia la seguridad, lo que corrobora el problema identificado en la presente investigación cuando se prioriza sólo el cumplimiento de un número de observaciones a realizar como meta del mes.

6. En el periodo de estudio, no se demostró que el cambio hacia los comportamientos seguros contribuyó a reducir los accidentes con incapacidad laboral, debido a la ocurrencia de accidentes después de la implementación del PSBC, se logró una reducción del $85.7 \%$ en la frecuencia de accidentes. Podría afirmarse que el periodo de estudio fue corto o que falto actualizar las tarjetas de observadores. También existen otros factores externos a considerar como la alta rotación de personal (retiro e incorporación de personal nuevo) o cambio de observadores o incorporación de nuevas líneas de producción.

\section{CONCLUSIONES}

1. Se encontró que la proporción de resultados de las observaciones de comportamientos seguros antes y después de las intervenciones con refuerzo positivo es diferente, es decir que la retroalimentación con refuerzo positivo influyó sobre los comportamientos seguros con una probabilidad de $95 \%$.

2. El promedio de accidentes con incapacidad laboral antes y después de la implantación del PSBC se reduce en un $85.7 \%$. 
3. En cuanto a la efectividad de la retroalimentación con refuerzo positivo realizadas al personal de producción, No hay diferencia significativa entre las medias de la Frecuencia de accidentes con incapacidad laboral antes y después de cada retroalimentación con refuerzo positivo, con una probabilidad de $95 \%$.

4. En el periodo de estudio, después de los dos primeros años de la implantación del Programa de seguridad basado en el comportamiento se logra reducir la ocurrencia de accidentes con incapacidad laboral, pero continuaron presentándose por lo que el periodo del estudio no fue suficiente para lograr la meta de cero accidentes.

\section{RECOMENDACIONES}

1. Para continuar con el éxito del Programa de seguridad basado en el comportamiento, la constante innovación en la gestión visual y el reconocimiento mantienen los resultados del programa.

2. El involucramiento de los líderes y del personal a su cargo es importante para enriquecer la cultura de seguridad de la planta.

3. En la capacitación de los observadores incluir el concepto del método $A B C$, para saber que activadores $\mathrm{o}$ antecedentes predisponen un comportamiento inseguro o saber cómo reforzar un comportamiento seguro a partir de sus consecuencias.

4. Replantear los comportamientos claves con el método DOIT para actualizar el diseño de las Tarjetas de observación.

5. El éxito de la seguridad basada en el comportamiento está en la medida que se hagan eficientemente las intervenciones con refuerzo positivo y no en la cantidad de observaciones realizadas para corregir los comportamientos inseguros.

\section{REFERENCIAS BIBLIOGRÁFICAS:}

[1] Alfaro, F. (2018). Estándar y Guía Técnica de Seguridad Conductual. Corporación nacional del cobre de Chile. p.32. Recuperado de: https:// www.academia.edu/5291334/Estándar_y_ Guía_Técnica_de_Seguridad_Conductual.

[2] De la Fuente, S. (2016), Aplicaciones de la Chi Cuadrado: Tablas de contingencia.
Homogeneidad. Dependencia e Independencia. Gestión Aeronáutica: Estadística Teórica. p.6. Recuperado de: http://www.fuenterrebollo. com/Aeronautica2016/contingencia.pdf

[3] Espluga, J. (2004). Actos inseguros en el trabajo: guía de intervención. NTP 415: Instituto Nacional de Seguridad e Higiene en el Trabajo (INSHT). Recuperado de https://www. insst.es/documents/94886/326962/ntp_415. pdf/ad4af595-e613-4ef0-ac08-ef0f58968349.

[4] Garlapati, A., Siddiqui, N. y Al-Shatti, F. (2013). Behavioral study of diverse workforce towards various Health, safety \& environment engagement strategies in upstream oil \& gas industries. International Journal of Scientific \& Engineering Research, 2 (5), 484 - 495. Recuperado de: https://ijser.org/researchpaper/ Behavioral-study-of-diverse-workforcetowards-various-Health-safety-environmentengagement-strategies-in-upstream-Oil-gasindustries.pdf

[5] Geller, E. S. (2005). Behavior-Based Safety and Occupational Risk Management. Behavior modification, 29 (3), p. 539-561, doi: 10.1177/0145445504273287.

[6] Hernández, R., Fernández, C. y Baptista, P. (2014). Metodología de la Investigación. México, D.F., México: McGraw-Hill.

[7] Jasiulewicz-Kaczmarek, M., Szwedzka, K. y Szczuka, M. (2015). Analysis of the Influence of the External Environment on the Development of Water Distribution System Exploitation Strategy - Case Study. Procedia Manufacturing, 3, p. 4876-4883. doi: 10.1016/j. promfg.2015.07.615.

[8] Ley $N^{\circ} 29783$ (20 de agosto del 2011). Ley de Seguridad y Salud en el Trabajo. El Peruano. Normas legales: 448694 - 448706.

[9] Martín, M. (mayo de 2014). Albert Ellis y el modelo ABC. En Rafael Santandreu (Presidencia), Escuela de Felicidad I Congreso, Barcelona, España. Recuperado de: https:// es.scribd.com/doc/ 280484652/Albert-Ellis-yEl-Modelo-ABC-Mar-Martin

[10] Martínez, C. (2014). El Proceso de Gestión de la Seguridad Basada en los Comportamientos: Actuación de los Supervisores en Empresas de Manufactura. (Tesis Doctoral). Universidad de León, España. http://hdl.handle. net/10612/5969

[11] Martínez, C. (2015). La gestión de la seguridad basada en los comportamientos: 
¿un proceso que funciona?. Medicina y Seguridad del Trabajo, 61(241), 424435. Recuperado de: http://scielo.isciii.es/ scielo.php?script=sci_arttext\&pid=S0465$546 \times 2015000400002 \&$ Ing $=e s \&$ tlng=es

[12] Meliá, J. L. (2007). Seguridad Basada en el Comportamiento. En Nogareda, C., Gracia, D.A, Martínez-Loza, J.F., Peiró, J.M., Duro, A., Salanova, M., Martinez, I:M:, Merino, J., Lahera, M., y Meliá, J.L.: Perspectivas de Intervención en Riesgos Psicosociales. Medidas Preventivas, p. 157-180. Recuperado de: https://www. insst.es/documents/94886/326962/ntp_415. pdf/ ad4af595-e613-4ef0-ac08-ef0f58968349

[13] Montero, R. (1999). Gestión de la seguridad basada en las conductas. Dirección $y$ organización: Revista de ingeniería de organización, 22, p. 85-93. Recuperado de: https://www.revistadyo.com/index.php/dyo/ article/view/280/280

[14] Montero, R. (2003). Siete principios de la Seguridad Basada en los Comportamientos. Prevención, Trabajo y Salud. (25), p. 4-11. Recuperado de http://www.insht.es/InshtWeb/ Contenidos/Documentacion/TextosOnline/Rev_ INSHT/2003/25/seccionTecTextCompl1.pdf.
[15] Montero, R (2010). Control de riesgos de accidentes y seguridad basada en Comportamientos. Zona Segura. 3(3), p. 2021. Recuperado de https://estrucplan.com.ar/ control-de-riesgos-de-accidentes-y-seguridadbasada-en-comportamientos/

[16] Pértega, S. y Pita, S. (2004). Asociación de variables cualitativas: El test exacto de Fisher y el test de McNemar. Cuadernos de atención primaria. 11, p. 304-308. Recuperado de http://www.agamfec.com/wp/wp-content/ uploads/2015/07/ 14_Invest_N11_5.pdf.

[17] Villalobos, C. (Febrero de 2018). Reconocimiento por logro de objetivos de seguridad en Planta Pucusana. En H. Núñez (Presidencia), Reunión de reconocimiento por logro de objetivos de seguridad $y$ producción del año 2017 en Planta Pucusana - Arcacontinental Lindley, Lima, Perú. 